\title{
Active Zones and the Readily Releasable Pool of Synaptic Vesicles at the Neuromuscular Junction of the Mouse
}

\author{
Rocío Ruiz, ${ }^{1}$ Raquel Cano, ${ }^{1}$ Juan José Casañas, ${ }^{1}$ Michael A. Gaffield, ${ }^{2}$ William J. Betz, ${ }^{2}$ and Lucía Tabares ${ }^{1}$ \\ ${ }^{1}$ Department of Medical Physiology and Biophysics, School of Medicine, University of Seville, 41009 Seville, Spain, and 2 Department of Physiology and \\ Biophysics, University of Colorado School of Medicine, Aurora, Colorado 80045
}

Synchronous neurotransmitter release is a highly regulated process that takes place at specializations at the presynaptic membrane called active zones (AZs). The relationships between AZs, quantal release, and vesicle replenishment are not well understood in a mature synapse. We have measured the number, distribution, and other properties of AZs in mouse motor nerve terminals and combined these observations with electrophysiological estimates of the size of the readily releasable pool (RRP) of synaptic vesicles. On average, we counted $850 \mathrm{AZs}$ per terminal. Assuming two primary docked vesicles per AZ, we predict a total of $\sim 1700$ vesicles optimally positioned for exocytosis. Electrophysiological estimates of the size of the RRP, using a simple kinetic model that assumes exponential depletion of the initial pool and refilling by recruitment, gave an average value of 1730 quanta during $100 \mathrm{~Hz}$ stimulation, in satisfying agreement with the morphology. At lower stimulus frequencies, however, the model revealed that the estimated RRP size is smaller, suggesting that not all AZs participate in release at low stimulation frequencies.

\section{Introduction}

Active zones (AZs) are sites in presynaptic nerve terminals that play a central role in neurotransmitter release (Couteaux and Pecot-Dechavassine, 1970; Landis et al., 1988; Zhai and Bellen, 2004). Each possesses a high density of voltage-gated calcium channels and other molecules that mediate the docking, fusion, and clearance of synaptic vesicles, the entire cycle requiring a few hundred milliseconds (Neher and Sakaba, 2008). Ultrastructural studies have revealed considerable variation in AZ structure in different species, and even within the same species between different synapses (for review, see Zhai and Bellen, 2004). Likewise, the number of AZs per terminal varies according to the size of the synapse, ranging from 1 or 2 in most central synapses to $\sim 600$ in the calyx of Held.

Both structural and functional studies have contributed to our understanding of AZs. In particular, ultrastructural studies of the adult mouse neuromuscular junction (NMJ) have revealed a consistent pattern, with each AZ hosting two docked vesicles at rest, with several additional nearby vesicles, some of which are docked (secondary vesicles), and others only tethered to AZ filaments (Nagwaney et al., 2009). Functional studies have allowed assessment of the behavior of synaptic vesicles in terminals in a variety of preparations. Of particular interest has been the calyx of Held, which can be dialyzed with a patch pipette, allowing, for example,

Received Sept. 3, 2010; revised Dec. 2, 2010; accepted Dec. 7, 2010.

This work was supported by Spanish Ministry of Education and Science Grants BFU2007-61171 (L.T.) and SAB2009-0022 (W.J.B.) and Muscular Dystrophy Association Grant MDA155642 (W.J.B., L.T.). R.C. is supported by Spanish Ministry of Education and Science Research Training Grant BES2008-005122.

Correspondence should be addressed to either of the following: Dr. William J. Betz, Department of Physiology and Biophysics, University of Colorado School of Medicine, Aurora, C0 80045, E-mail: bill.betz@ucdenver.edu; or Dr. Lucía Tabares, Department of Medical Physiology and Biophysics, School of Medicine, University of Seville, 41009 Seville,Spain, E-mail: Itabares@us.es.

DOI:10.1523/JNEUROSCI.4663-10.2011

Copyright $\odot 2011$ the authors $\quad 0270-6474 / 11 / 312000-09 \$ 15.00 / 0$ detailed biophysical measures of the calcium sensitivity of various stages of exocytosis and recovery, and the characterization of vesicle pool dynamics (Neher and Sakaba, 2008). It has been suggested that, after a vesicle in the readily releasable pool (RRP) undergoes exocytosis, it is replaced by one of the nearby vesicles, a process called "positional priming" in the calyx (Wadel et al., 2007; Neher and Sakaba, 2008).

Although most attempts to characterize the RRP have begun with electrophysiological observations of evoked transmitter release, we began instead from a morphological perspective, estimating RRP size by counting the total number of AZs in mouse motor terminals. We then turned to electrophysiology to see whether we could functionally identify such a population as readily releasable. The ability of a simple kinetic model of RRP depletion and its replenishment to fit evoked endplate potential (EPP) rundown during repetitive stimulation was good, and so we explored additional properties of the RRP and its replenishment. For example, we show that the fits of quantal loss from and recruitment to the RRP during repetitive stimulation are normally tightly coupled over a 100 -fold range of stimulus frequency, although the evident coupling can be disrupted by temperature, EGTA, or phorbol ester (PE). In addition, we show that the RRP size estimated from the model is frequency dependent, more than doubling as stimulation frequency increases from 1 to $100 \mathrm{~Hz}$, suggesting that, at low frequencies, many AZs are apparently silent. The model involves depletion of the RRP during a train following an exponential time course, leaving vacant release sites that are reoccupied by new, or recycled, vesicles, and is compatible with the positional priming model (Wadel et al., 2007; Neher and Sakaba, 2008).

\section{Materials and Methods}

Muscle preparation. Mice of either sex were killed by means of $\mathrm{CO}_{2}$. The levator auris longus muscle was dissected with its nerve branches intact 
and pinned to the bottom of a $2 \mathrm{ml}$ chamber, over a bed of cured silicone rubber (Sylgard; Dow Corning). Preparations were continuously superfused with a physiological solution containing the following (in $\mathrm{mM}$ ): 125 $\mathrm{NaCl}, 5 \mathrm{KCl}, 2 \mathrm{CaCl}_{2}, 1 \mathrm{MgCl}_{2}, 25 \mathrm{NaHCO}_{3}$, and 30 glucose, continuously gassed with $95 \% \mathrm{O}_{2}$ and $5 \% \mathrm{CO}_{2}, \mathrm{pH} 7.35$. Recordings were performed at room temperature $\left(22-23^{\circ} \mathrm{C}\right.$ ) or (see Fig. $7 \mathrm{~A}$ ) at $36 \pm 1{ }^{\circ} \mathrm{C}$ using a dish heater DH-35 and inline heater SF-28 controlled and monitored by a dual automatic temperature controller TC-344B, all from Warner Instruments.

Electrophysiology. The nerve was stimulated by means of a suction electrode. The stimulation consisted of square-wave pulses of $0.2 \mathrm{~ms}$ duration and 2-40 V amplitude, at variable frequencies $(0.5-100 \mathrm{~Hz})$. EPPs and miniature EPPs (mEPPs) were recorded as described previously (Ruiz et al., 2008). Muscle contractions were blocked by $\mu$-conotoxin GIIIB (Alomone Labs; $2-4 \mu \mathrm{M}$ ), a specific blocker of skeletal muscle voltage-gated sodium channels, except in Figure 7A, where 1.1 $\mu \mathrm{M}$ curare was used (and thus EPPs were not converted to quantum contents).

Quantum contents during a train were plotted against time and fitted to a model that assumed that all release on the first shock came from the RRP, which subsequently was depleted along an exponential time course. To account for the plateau phase, we assumed that a recruitment process began some time after the first shock and rose sigmoidally to the plateau level as the original RRP became depleted. Although we fit our data to this single model, we explored several different mathematical expressions to fit the sigmoid portion of the curve, with indistinguishable results. These included the following: a standard sigmoid shape $(C /(1+\exp (-(D-$ $t) / E))$ ), where $C$ represents amplitude; $D$, half-time of rise; and $E$, steepness of rise of the sigmoid. This had the disadvantage that the sigmoid was not constrained to start at zero. We also used an exponential raised to a power: $C^{\star}(1-\exp (-t / D))^{\mathrm{E}}$, and a rising exponential with a delayed start time: $C^{\star}(1-\exp (-(t-D) / E))$, where $D$ represents start time of the exponential rise (in this case, $Y$ values were constrained to be $\geq 0$ ). Each technique required five variables (two for the decaying exponential and three for the sigmoid). With the last technique, we could obtain good results with four variables (making the time constants of both exponentials the same), except that four variables could not account for the transient rise that occurred in some preparations after the initial rundown.

The iterative model calculations (see Fig. 8) were performed as follows: a binary vector equal in size to the RRP (the number of available release sites) (see Fig. 6E) was created. Each site was updated after every stimulus in terms of its occupancy (ability to release a quantum). After release occurred from a site, it was not reoccupied until a "dead time" had elapsed. During each run (one train of 100 stimuli), the dead time was constant and, in Figure $8 \mathrm{~A}$, was equal to the half-time of the sigmoidal rise in EPP recruitment during a train (see Fig. $6 C$ ). For Figure $8 B$, dead time was varied systematically between runs from 0 to $10 \mathrm{~s}$ in increments of $5 \mathrm{~ms}$ (thus, for each stimulus frequency, 2000 runs were made, each with a different value of recruitment time). For each stimulus in a run, the number of occupied sites $n$ and the observed quantum content $m$ were used to calculate release probability $p$. The value of $n$ was then updated, being reduced by $m$ and increased by the number of sites that had been unoccupied for longer than the recruitment time. This iteration was repeated for each stimulus to the end of the train, at which time the overall (root mean square) variance in $p$ was calculated as the difference between the $p$ value of the first stimulus $\left(p_{1}\right)$ and every other $p$ value, viz, $\operatorname{sqrt}\left(\sum\left(p-p_{1}\right)^{2}\right)$. It was this number that we sought to minimize by repeating the procedure with a range of different recruitment times $(0-10 \mathrm{~s}$ in increments of $5 \mathrm{~ms})$. The entire procedure was then repeated for each frequency.

Immunohistochemistry. Whole-mount dissected muscles were incubated for 30 min in Ringer's solution saturated with $95 \% \mathrm{O}_{2} / 5 \% \mathrm{CO}_{2}$ before fixation in $4 \%$ paraformaldehyde. Later, muscles were bathed in $0.1 \mathrm{M}$ glycine in PBS for $20 \mathrm{~min}$, then permeabilized with $1 \%(\mathrm{v} / \mathrm{v})$ Triton $\mathrm{X}-100$ in PBS for $30 \mathrm{~min}$ and incubated in 5\% (w/v) BSA, 1\% Triton $\mathrm{X}-100$ in PBS for $2 \mathrm{~h}$. Samples were incubated overnight at $4^{\circ} \mathrm{C}$ with the primary antibodies of interest (see below). The following day, muscles were rinsed for $1 \mathrm{~h}$ in PBS containing 1\% Triton X-100, incubated for $1 \mathrm{~h}$ both with the corresponding secondary antibodies and $10 \mathrm{ng} / \mathrm{ml}$ rhodamine-BTX (Invitrogen), and rinsed again with PBS for $90 \mathrm{~min}$.
Finally, muscles were mounted in glycerol containing DABCO (diazabicyclo[2.2.2] octane).

To detect the AZs, we used a mouse monoclonal antibody against Bassoon (Stressgen) and a rabbit polyclonal against Piccolo (Synaptic Systems). The secondary antibodies were goat anti-mouse and donkey anti-rabbit Alexa 488, Alexa 594, and Alexa 647 (Invitrogen).

Some preparations were treated with collagenase type I (SigmaAldrich; $0.1 \%$ ) for $30 \mathrm{~min}$ followed by protease (Sigma-Aldrich; $0.01 \%$ ) for $30 \mathrm{~min}$, both in the presence of curare (Sigma-Aldrich; $5 \mu \mathrm{g} / \mathrm{ml}$ ) to block contractions, and then prepared for immunostaining as described above.

Image acquisition and analysis. Muscles were imaged with an upright Olympus FV1000 confocal laser-scanning microscope, equipped with three excitation laser lines (argon-krypton laser with 488, 561, and 633 $\mathrm{nm}$ excitation lines). Emission colors were detected sequentially. Images were taken using a $63 \times$ oil-immersion objective with a numerical aperture of 1.4.

In doubly stained (Bassoon and Piccolo) preparations, the overlap between the two was incomplete. This probably reflects interference between the antibodies because, for example, the number of spots in Piccolo-only preparations was higher than in preparations stained simultaneously with Bassoon and Piccolo. Thus, in the quantifications reported, we used mainly preparations stained only with Bassoon. We focused in particular on those preparations that revealed crisp spots and discarded preparations in which the staining was more diffuse. Terminals were sought that lay on the superficial surface of surface muscle fibers, providing a clear en face view of the entire terminal. Terminal sizes were determined automatically (by opening, then closing) to generate the region of interest or manually (by tracing the outline) from Bassoonlabeled terminals.

For detection and measurement of Bassoon and Piccolo spots, we used ImageJ and custom routines written in Igor or Matlab. For Gaussian fitting of spots, we used the following procedure: the image was heavily smoothed (moving bin average of 11-21 pixels) and the outline mask was determined by brightness thresholding. The original image was then lightly smoothed (moving bin of 3-5 pixels), the mask was applied, and the watershed function was run. The spots for additional analysis were then selected by a combination of criteria, including spot size, threshold brightness, and finally by manual selection or rejection (the manual process was applied to only a few percent of the total number). Each selected spot was then analyzed in two ways. First, a two-dimensional Gaussian was fitted to the spot. In addition, the brightest pixel was found, and all contiguous pixels that exceeded a certain threshold (usually $70 \%$ of the difference between the brightest and dimmest pixels) were included in the spot. The results of both measurements agreed well.

All statistics are given as mean \pm SEM, unless stated otherwise.

\section{Results}

\section{Counting active zones in motor nerve terminals}

Figure $1 A$ shows an example of a terminal fixed and labeled with antibodies against Bassoon, a scaffolding protein of AZs (tom Dieck et al., 1998). The pattern of crisp fluorescent spots is similar to that reported previously (Nishimune et al., 2004). The boxed region is enlarged in the bottom panels, which illustrate our method of identifying and measuring various properties of each spot. Watershed segmentation outlined the regions (left), which were then usually selected (blue) or deselected (magenta) automatically according to several criteria (see Materials and Methods). The selected regions were then analyzed (see Materials and Methods), the calculations producing the spots on the right (white outline). These spots were then best fit with a two-dimensional Gaussian, from which measurements like those shown in Figure $1 C-E$ are presented. The Gaussian fits were good, with $95 \%$ of $R^{2}$ values exceeding 0.8 (results not shown).

The total number of Bassoon spots per terminal varied with the size of the terminal, averaging $850 \pm 252 \mathrm{AZs} /$ terminal $($ mean $\pm \mathrm{SD})($ Fig. $1 \mathrm{~B})$. We also measured the area of presynaptic 

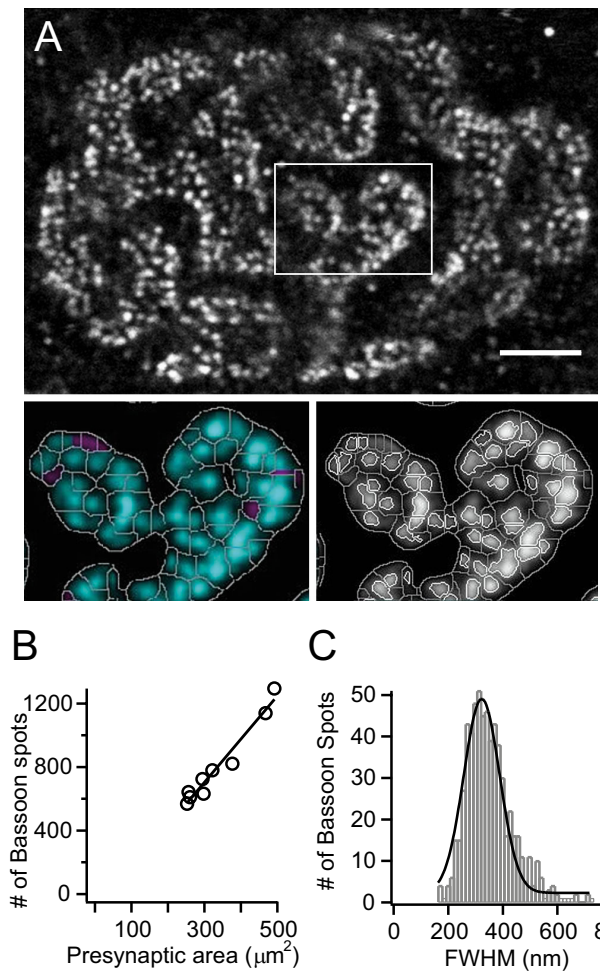

C

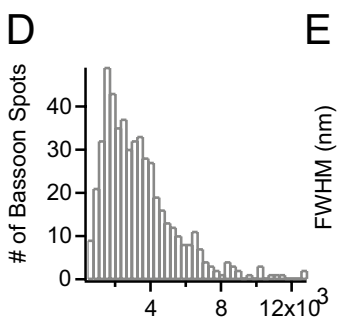

Integrated Fluorescence (a.u.

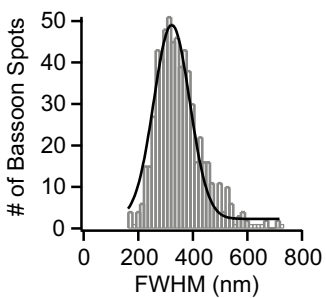

$\mathrm{E}$

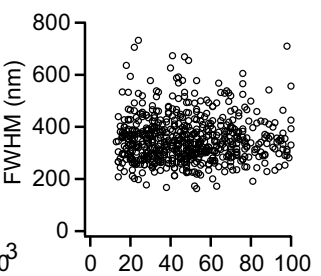

Max. Fluorescence (a.u.)

Figure 1. Characteristics of fluorescent spots labeled with anti-Bassoon antibodies. $\boldsymbol{A}$, Single confocal section of a NMJ labeled with anti-Bassoon-Alexa 647. Bassoon spots are distributed throughout the terminal. The region in the rectangle is enlarged below and illustrates the segmentation method using a watershed function to identify spots (left panel), which are then selected (blue) or deselected (magenta) according to spot size and brightness. Selected spots are then further segmented (right panel; white lines) (see Materials and Methods). Scale bar, 5 $\mu \mathrm{m}$. $\boldsymbol{B}$, The mean number of Bassoon spots per terminal is proportional to the terminal size (mean number of particles, $850 \pm 252 ; n=9$; mean \pm SD). $C$, Distribution of FWHMs of Bassoon spots from terminal in $\boldsymbol{A}$. D, Distribution of the total brightness (integrated fluorescence) of Bassoon spots from a Z-projection from a different terminal. $\boldsymbol{E}$, Relationship between maximum fluorescence values of each spot with the half-width of the spots, from terminal shown in $\boldsymbol{A}$, showing a poor correlation between these two parameters.

terminals, which averaged $335.9 \pm 90.5 \mu \mathrm{m}^{2}(n=9)$, yielding a density of $2.37 \pm 0.16 \mathrm{AZs} / \mu \mathrm{m}^{2}$, in good agreement with estimates from freeze fracture results (Fukunaga et al., 1983). The average diameter [full width half-maximum (FWHM)] (Fig. 1C) of the spots in different preparations varied from $\sim 260$ to 350 $\mathrm{nm}$, and the distribution of diameters was well fit by a Gaussian, with a small tail at larger diameters. This average diameter was close to that observed with 200-nm-diameter (diffractionlimited) beads (results not shown), suggesting that the Bassoon spots were themselves diffraction limited, consistent with ultrastructural measurements of AZ sizes (Fukuoka et al., 1987) $(80 \times$ $60 \mathrm{~nm}$ ). The distribution of total brightness (integrated fluorescence) showed a more skewed distribution (Fig. 1D). The tail may reflect the occasional AZ clusters observed in freeze fracture EM (Fukunaga et al., 1983; Teng et al., 1999) and EM tomogra- phy (Nagwaney et al., 2009). We examined the locations of the Bassoon spots with the largest integrated fluorescence values and found that they were scattered over the entire terminal (results not shown) (see Discussion). Finally, there was no correlation between spot size (FWHM) and maximum fluorescence (Fig. $1 E)$. Similar results to these, and all other results with Bassoon antibodies, were obtained using an antibody against Piccolo, another AZ scaffolding protein (Cases-Langhoff et al., 1996; Fenster et al., 2000) (results not shown).

The spatial distribution of the Bassoon spots appeared quite regular by eye (Fig. 1A); signs of clustering, for example, were rare, and spots sometimes could be seen to line up across the width of the terminal (Fig. 2A). This regular spacing of AZs was confirmed in nearest neighbor analyses performed on whole terminals. Figure $2 B$, left, shows that the Bassoon spots (centers of two-dimensional Gaussian fits) were regularly spaced (average nearest neighbor separation $528 \pm 3 \mathrm{~nm}$; mean $\pm \mathrm{SEM} ; n=7$ terminals); similar results were obtained with Piccolo (438 \pm 9 $\mathrm{nm} ; 2$ terminals). We compared these observed distributions with simulated ones (Fig. $2 B$, right panel), generated by randomly placing an equal number of 250 -nm-diameter spots (without overlap) and measuring nearest neighbors (the simulation was repeated 100 times, and the results averaged). The resulting distribution peaked at the minimum separation, as expected, and was clearly different from the observed ( $p<0.0001 ; \chi^{2}$ test).

A ready explanation for the nonrandom spacing of the Bassoon spots was evident when we compared Bassoon spot positions with the locations of postsynaptic folds, identified by labeling acetylcholine (ACh) receptors with rhodamineconjugated $\alpha$-bungarotoxin (BTX-Rho), as shown in Figure 2C (central panel). Bassoon spots (left panel) were segmented as described above; spot centers are marked with red dots in the Bassoon image (left panel) and in the BTX-Rho image (right panel). With few exceptions, every Bassoon spot center lay over a postsynaptic fold, as expected for AZs (Heuser et al., 1974; Rash and Ellisman, 1974; Nagwaney et al., 2009).

We looked for ways to disrupt the uniform Bassoon staining. We wondered, for example, whether intense stimulation would fragment or otherwise disrupt the spots, and tested this by stimulating massive exocytosis with elevated potassium ion concentration (70 mm for $1 \mathrm{~min}$ ) before fixation. As shown in Figure 3A, Bassoon labeling after stimulation was indistinguishable from control (unstimulated) terminals, suggesting that at this resolution the AZs, each of which on average played host to tens of vesicles undergoing exocytosis during the stimulation period, are very stable. In the frog, Heuser et al. (1979) showed that AZs were not altered by intense stimulation (4AP). Ceccarelli et al. (1979) showed that low calcium (for 2 h, plus EGTA) disrupted AZs severely, and it was not reversible. Treatment with proteolytic enzymes (collagenase followed by protease), however, which has been shown to disrupt the normal AZ pattern in freeze fracture EM (Nystrom and Ko, 1988), disrupted the pattern of Bassoon staining (Fig. 3B). The images after enzyme treatment were quite variable, but in general the Bassoon staining was much diminished (left-hand panel). Matched controls (right-hand panel) were uniformly consistent in their pattern. Thus, it is clear that proteolytic enzymes disrupt the pattern of immunostaining. These results support the idea that the Bassoon spots are indeed marking locations of AZs.

\section{Physiological correlates of docked vesicles}

With $\sim 850 \mathrm{AZs}$ per terminal, we expect $\sim 1700$ docked and primed vesicles at each resting synapse, and several additional 

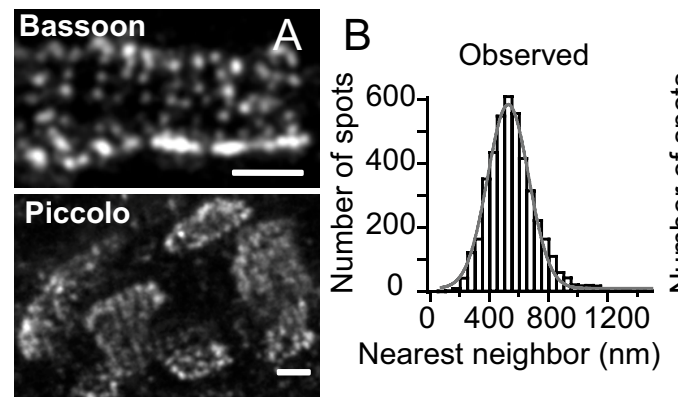

Nearest neighbor $(\mathrm{nm})$
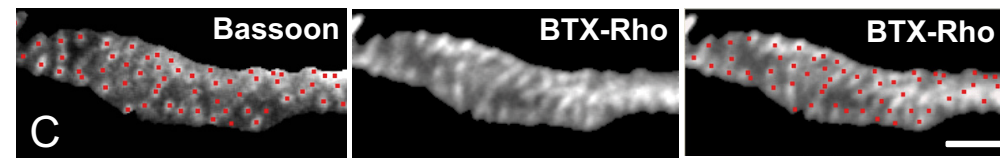

Figure 2. Spatial pattern of Bassoon and Piccolo spots. A, Top panel, Z-projection of part of an anti-Bassoon-labeled terminal. Some spots are clearly aligned in parallel lines that run perpendicular to the long axis of the nerve terminal. Bottom panel, Terminal stained for Piccolo. B, Observed (left) nearest neighbor distribution of Bassoon spots in a representative terminal is well fit by a Gaussian. The mean \pm SD nearest neighbor separation is $532 \pm 260 \mathrm{~nm}$. In a simulation (right), the same number of spots placed randomly in the terminal gave a very different distribution $(p<0.0001)$. C, Left panel, High-magnification of Bassoon spots showing the centroid (red squares) of each spot. Middle, The postsynaptic membrane stained with BTX-Rho shows regions of high intensity corresponding to the openings of postsynaptic folds. Right, Bassoon centroids lie precisely over postsynaptic folds. Scale bars, $2 \mu \mathrm{m}$.

vesicles tethered nearby (Nagwaney et al., 2009). We looked for functional signs of these morphologically defined populations of vesicles, expecting that the primary docked vesicles would be the first ones to be released with repetitive nerve stimulation, thereby defining the RRP functionally. A typical recording of EPPs during $100 \mathrm{~Hz}$ stimulation is shown in Figure $4 A$. Recordings were obtained without blocking postsynaptic ACh receptors (muscle twitches were blocked by $\mu$-conotoxin). We calculated quantum content $(m)$ for each EPP by correcting its observed peak amplitude for nonlinear summation of voltage (McLachlan and Martin, 1981) and dividing by the mean amplitude of mEPPs; typical results are illustrated in Figure $4 B$ (average of three trains, from a total of 40 control fibers from seven muscles) and show that quantum contents fell progressively from an initial value to a plateau that was approximately onehalf the initial amplitude, as has been observed many times (Elmqvist and Quastel, 1965; Zucker and Regehr, 2002).

To estimate the size of the RRP, two different techniques have been used. In one (Fig. 4C), the cumulative quantum content is plotted against time, and a back extrapolation of the linear part of the curve to the $y$-axis (straight line), corresponding to the plateau phase of the recording, gives a measure that is sometimes taken as the RRP (Schneggenburger et al., 1999; Millar et al., 2002; Stevens and Williams, 2007; Pan and Zucker, 2009), in this case $\sim 500$ quanta, less than one-third of the prediction from morphology. However, a problem exists with this technique if EPP amplitudes during the plateau do not decline to zero (i.e., the cumulative quantum content trace fails to become flat). This is because the method assumes that release from the pool responsible for the plateau is present from the beginning of the train. The back extrapolation then gives the size only of the initial component that rides on top of the plateau. In other words, this method assumes a "parallel model" for vesicle release, with release even on the first shock coming from two pools, one that declines quickly to zero (taken as the RRP), and one that is constant throughout the train. In a recent comprehensive model of transmitter release (Pan and Zucker, 2009), it was not possible to identify a specific vesicular pool with this technique. Because EPP amplitudes in our experiments never fell to zero during the plateau, we rejected this approach.
A second procedure (Fig. 4D) assumes a "sequential model," namely that initially all of the vesicles come from one pool (the $\mathrm{RRP})$ and that as this pool is depleted (exponentially, ignoring the initial facilitation), a process of recruitment begins, increases, and eventually maintains the plateau at a reasonably steady level (Elmqvist and Quastel, 1965). The RRP size can be estimated by plotting quantum content ( $y$-axis) against accumulated quantum content (Fig. 4D) and drawing a straight line through the declining phase; its $x$-axis intercept $(\sim 2000)$ gives an estimate of the RRP size. As expected, when we used this method we obtained significantly higher values than given by the back-extrapolation technique of Figure $4 C$, values within the range determined from the morphological estimation of the number of release sites.

As illustrated in Figure $4 E$, we devised a simple variation of the second technique, which includes also a quantitative estimate of the kinetic properties of the recruitment process. Specifically, we fit the entire observed curve of quantal content rundown (except for the initial facilitation, which lasted 0-4 shocks, and led to an overestimate of RRP size by $0-3 \%$ ) with two functions: a declining exponential (the contribution to each EPP from vesicles docked at the start of the train) plus a rising sigmoid, representing the contribution to each EPP from recruited vesicles. We explored several different functions to describe the sigmoid (see Materials and Methods), which were equally capable in fitting results, for example, $m(t)=A^{*} \exp (-t / B)+C /(1+$ $\exp (-(t-D) / E))$, where $m$ represents quantum content; $A$, initial $m ; B$, time constant of RRP depletion; $C$, mean amplitude of the plateau; $D$, half-time of refilling; and $E$, steepness of the refilling time course. The integration of the first exponential gives the size of the RRP.

The average size of the RRP calculated in this way was $1730 \pm$ 55 quanta, in satisfying agreement with our morphological observations (1700 primary docked vesicles). According to this sequential model, during the first $\sim 200 \mathrm{~ms}$ of a $100 \mathrm{~Hz}$ train, $\sim 90 \%$ of the quanta come from the RRP, and after 40-50 stimuli at 100 $\mathrm{Hz}$ virtually all the quanta from the RRP have been released.

With five unconstrained variables, the good quality of the fit to the observed data is perhaps not surprising, but an additional observation lent further support to the model. In some, but not all trains, the EPP amplitudes did not decay to the plateau monotonically. Instead, the curve declined for $\sim 50 \mathrm{~ms}$, then rose again transiently, before settling down to the plateau level (see Fig. 7B). Such biphasic behavior is readily explained by a recruitment process that, although often exquisitely matched to the loss of the RRP, sometimes produces a small rebound, or extra refilling of the vacated AZ sites. This behavior was especially noticeable in human muscle (Elmqvist and Quastel, 1965).

\section{Recovery of the RRP}

To study the refilling of the RRP, we gave pairs of trains, with a delay of $\sim 0.1-6 \mathrm{~s}$ between the first and second train (pairs were separated by $1 \mathrm{~min}$ ). We then fitted each curve as described above and determined the size of the RRP. RRP sizes in response to the first (control) train of each pair, on average, decreased slightly 

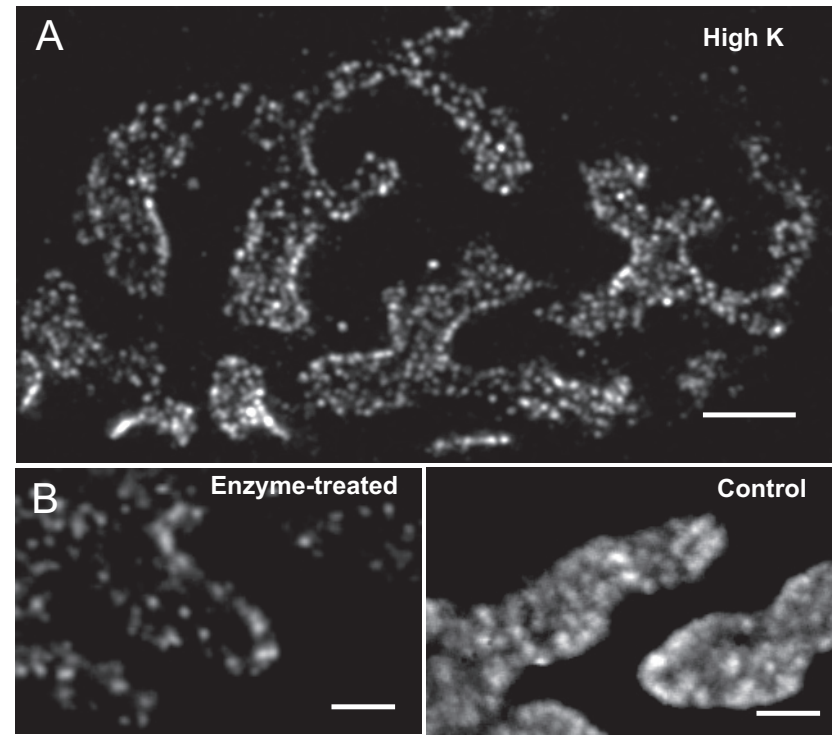

Figure 3. Bassoon spots under perturbing conditions. $A$, Stimulation with $70 \mathrm{~mm} \mathrm{~K}^{+}(1 \mathrm{~min}$ duration) did not disrupt the pattern of Bassoon staining, which is indistinguishable from controls. Scale bar, $5 \mu \mathrm{m}$. $\boldsymbol{B}$, Left, Exposure to proteolytic enzymes (collagenase plus protease for $30 \mathrm{~min}$ ) severely disrupted the amount and pattern of Bassoon staining. Right, Control terminal processed in parallel. Scale bars, $2 \mu \mathrm{m}$.
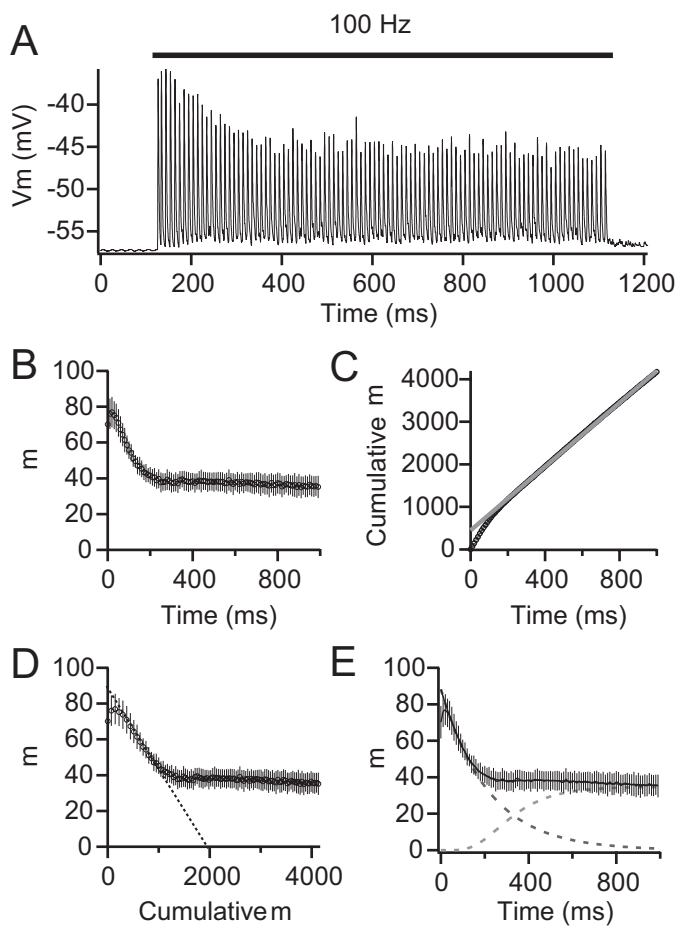

Figure 4. Dynamics of neurotransmitter release and calculation of RRP size. $\boldsymbol{A}$, Peak endplate potential amplitudes during a $100 \mathrm{~Hz}, 1 \mathrm{~s}$, train of stimulation show a characteristic decay to a plateau. $B$, Quantum contents $(m)$ during the train (mean \pm SEM for 3 sequential trains). $C$, Estimating RRP size by plotting cumulative $m$ versus time, and linearly extrapolating to the $y$-axis (see text for critique). D, Estimating RRP size by plotting $m$ ( $y$-axis) versus cumulative $m$ ( $x$-axis) and linearly extrapolating the initial decline to the $x$-axis. $E$, Estimating RRP size and recruitment time by curve fitting to a function of the sum of exponential decay of the RRP plus sigmoidal rise of recruitment (dotted lines). Methods $\boldsymbol{D}$ and $\boldsymbol{E}$ ignore contributions from facilitation.

over time ( $\sim 14 \%$ after the tenth pair of trains) (data not shown). The ratio of the RRP of the second train divided by the RRP of the first train in each pair is plotted against intertrain interval in Figure 5. The best fit exponential time constant is $823 \mathrm{~ms}$. This is

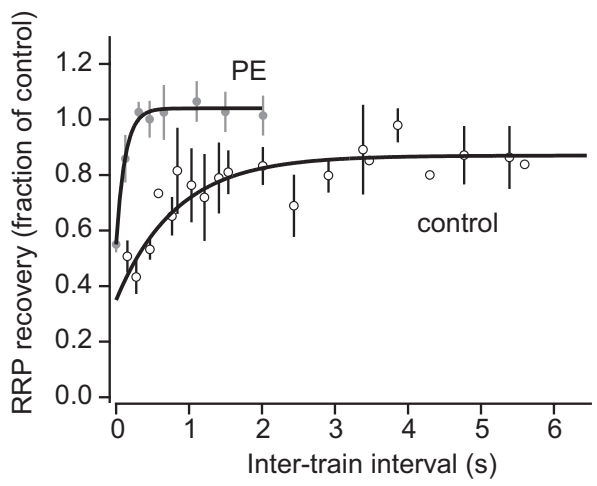

Figure 5. RRP recovery time course. Paired trains (each $100 \mathrm{~Hz}$ for $1 \mathrm{~s}$ ) were given, separated by varying intervals. The size of the RRP was measured for each train, and the ratio (second train/first train) is plotted against intertrain interval. The best exponential fit to control preparations is $823 \mathrm{~ms}$, with recovery reaching $87 \%$ of the control value. Also shown is the effect of treatment with $\mathrm{PE}(1 \mu \mathrm{M})$, which greatly accelerated recovery.

somewhat slower than the half-time of recruitment during a train $(\sim 280 \mathrm{~ms})$ and likely reflects the rapid fall in intracellular calcium concentration that occurs immediately after the train (David and Barrett, 2000). The average recovery is to $87 \%$ of the control level, which suggests that a small component of the recovery is slower than several seconds. Also shown in Figure 5 is the effect of phorbol ester (described below).

\section{Effect of stimulus frequency}

We used this model to examine the effects of several experimental manipulations. Figure $6 \mathrm{~A}$ shows EPP amplitudes (normalized) during trains of 100 shocks at frequencies of $1,5,10,20,50$, and $100 \mathrm{~Hz}$ (average results from four fibers). At all frequencies, the EPP amplitudes declined to a flat plateau, which was lower with higher frequencies. The plateau level (relative to the initial level) is plotted in Figure $6 B$, which shows a steep decline in the plateau as the frequency rose to $10 \mathrm{~Hz}$, but then a leveling off at higher frequencies. Figure $6 \mathrm{C}$ shows three of the trains and their associated best fits to the model of RRP depletion and refilling described above. The tight coupling between RRP depletion and recruitment over this 100 -fold range of frequencies is evident from the fitted curves, and Figure $6 D$ shows the close agreement between the two. Finally, Figure $6 E$ shows that the apparent size of the RRP (the integral of the fitted exponential) was not constant with frequency of stimulation, but more than doubled as the frequency increased from 1 to $100 \mathrm{~Hz}$. One interpretation of this result is that not all AZs are functional at low stimulus frequencies, but all are recruited during high-frequency stimulation. Thus, the first shock in any train at any frequency will draw from a small subset of AZs, and then, if the frequency is high, subsequent stimuli will awaken the other AZs, presumably because of the rise in global calcium, the rate of which is rapid (David and Barrett, 2000). Our estimates of RRP size, for example, are determined primarily by the first $150-200 \mathrm{~ms}$ of a train at $100 \mathrm{~Hz}$ (Fig. $6 \mathrm{~A}$ ). Perhaps the facilitation that can be seen during the first few shocks with high-frequency stimulation reflects in part this awakening of sleepy AZs.

\section{Altering the coupling between RRP depletion and recruitment}

We used our model to examine the effects of several experimental manipulations. Temperature had a clear effect. We compared $\mathrm{RRP}$ rundowns at 23 and $37^{\circ} \mathrm{C}$ (Fig. $7 \mathrm{~A}$ ). The initial amplitude of 


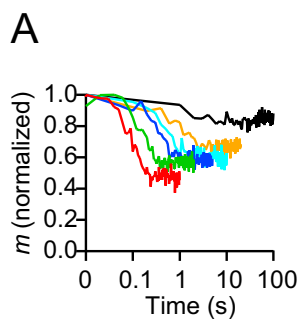

B
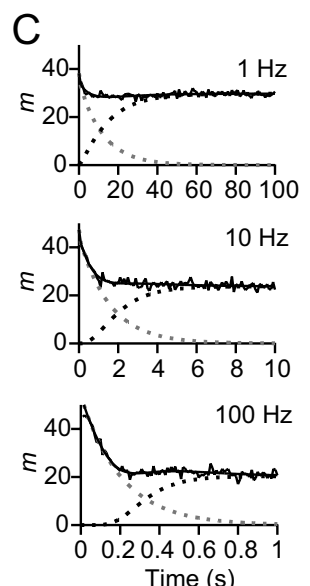

Time (s)
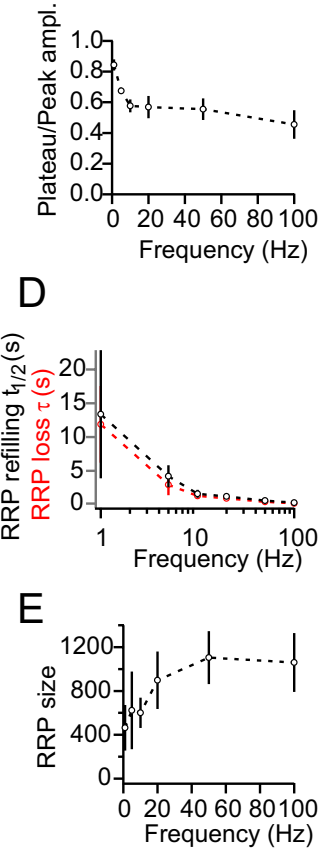

Figure 6. Frequency dependence of RRP size, depletion, and refilling. $\boldsymbol{A}$, Quantum content of EPPs during trains of different frequencies [1 (black), 5 (yellow), 10 (light blue), 20 (dark blue), 50 (green), and 100 (red) Hz], each with 100 shocks, as a fraction of the largest EPP in the train. Logarithmic $x$-axis. $\boldsymbol{B}$, Relative plateau values as a function of stimulation frequency. The plateau level declines steeply between 1 and $10 \mathrm{~Hz}$, and then levels off, changing relatively little between 10 and $100 \mathrm{~Hz}$. C, Three examples of quantum contents (1, 10, and $100 \mathrm{~Hz}$ trains) illustrating the tight coupling between RRP loss and recruitment, over a 100-fold range of frequency. Note the changing $x$-axis limits. $D$, The time constant of RRP loss (red) and the half-time of RRP refilling (black) are steeply dependent on stimulation frequency ( $x$-axis) but tightly coupled. Logarithmic $x$-axis. $E$, Calculated RRP size depends on stimulation frequency, more than doubling as the frequency rises from 1 to $100 \mathrm{~Hz}$. Error bars are $\pm 1 S D$.

EPPs was not significantly altered by changing the temperature, nor was the initial rate of EPP decline during $100 \mathrm{~Hz}$ stimulation, which meant that the RRP size was unaltered. The plateau level, however, was higher at $37^{\circ} \mathrm{C}$, which the model interprets as an acceleration of recruitment, consistent with the observation that synaptic vesicle mobility increases steeply with temperature (Gaffield and Betz, 2007), despite the observation that global calcium concentration is lower at 32 than at $18^{\circ} \mathrm{C}$ (David and Barrett, 2000).

PE, which directly activates protein kinase C (Shapira et al., 1987; Waters and Smith, 2000) and Munc-13 (Betz et al., 1998; Rhee et al., 2002), has been shown to potentiate neurotransmission in different synapses, including the NMJ (Ruiz et al., 2008) and the calyx of Held (Hori et al., 1999). We tested the effects of 4- $\beta$-phorbol-12,13-dibutyrate $(1 \mu \mathrm{M})$ at the mouse NMJ and found that it increased quantum content during low-frequency stimulation but, as shown in Figure $7 B$, did not significantly alter RRP size. The EPP plateau was significantly higher, and a clear transient "bump" appeared at the beginning of the plateau, reflecting an increase in recruitment rate. In addition, as shown in Figure 5, the RRP recovery rate after the end of the train was accelerated.

Global calcium has been implicated in recruitment rate, and application of $30 \mu \mathrm{M}$ EGTA-AM for $30 \mathrm{~min}$ reduced both initial EPP amplitude and the plateau level during $100 \mathrm{~Hz}$ stimulation (Fig. 7C, left panel). The plateau reduction was relatively greater,
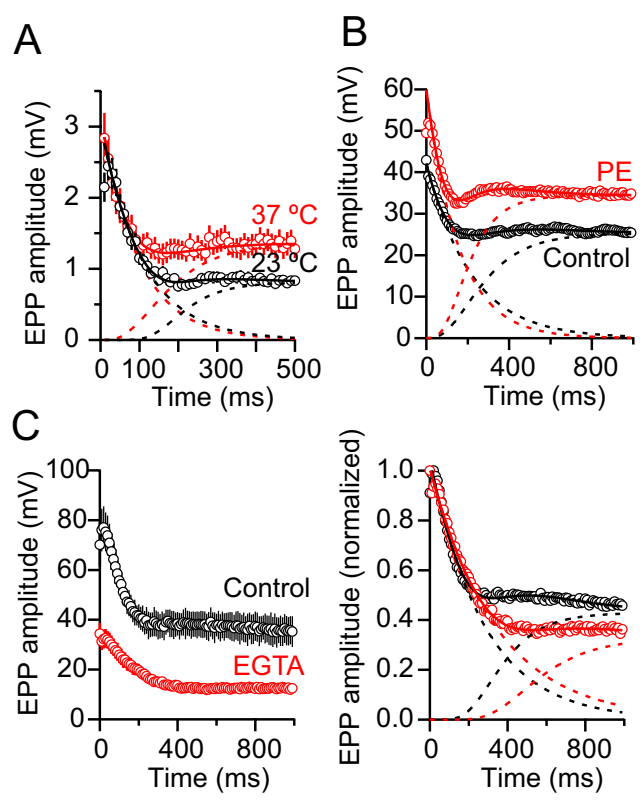

Figure 7. Disrupting the coupling between RRP depletion and recruitment. $\boldsymbol{A}$, Raising the temperature from $23^{\circ} \mathrm{C}$ (black) to $37^{\circ} \mathrm{C}$ (red) did not change the RRP size (decaying red and black are nearly identical) but shortened the half-time of recruitment (sigmoidal dashed lines). Muscle contraction was blocked with $1.1 \mu \mathrm{m}$ curare in this experiment. $\boldsymbol{B}, \mathrm{PE}(1 \mu \mathrm{m})$ increased initial $m$ (from 44 to 64 quanta) but did not significantly change RRP size (control, 950; PE, 997). RRP rundown was faster (control, $218 \mathrm{~ms} ; \mathrm{PE}, 156 \mathrm{~ms}$ ) and the half-time of the rise in recruitment was shorter (control, $280 \mathrm{~ms}$; PE, $210 \mathrm{~ms}$ ). PE also greatly accelerated the recovery of the RRP after the end of the train (Fig. 5,PE). C, Treatment with EGTA-AM reduced quantum content (left panel) and RRP size. When normalized to initial quantum contents (right panel), it is clear that EGTA treatment selectively decreased the plateau, which according to the kinetic model reflects slowed recruitment.

however (Fig. 7C, right panel), and the model fitting suggested that the recruitment rate had been reduced by the EGTA.

\section{Iterative model of recruitment during stimulation}

The good agreement between the morphological and physiological estimates of RRP size encouraged us to examine the kinetic model in more detail. The model divides release into two components for each EPP: quanta that arise from vesicles that were docked at the membrane when the train started (the decaying exponential), and quanta that arise from vesicles that were recruited to release sites after the train started (the rising sigmoid). We wondered whether the shape of the rising sigmoid could inform us about underlying rates of vesicle recruitment. In particular, we would like to know how much time elapses after exocytosis of a vesicle before that site is refilled. We refer to this as the dead time that follows exocytosis. If we knew the dead time, we could calculate the number of release-ready sites at any time, and therefore we could calculate release probability $p$. Unfortunately, we know neither the dead time or $p$, and the sigmoid shape is of no help, for it informs us only of quanta released, not releaseready vesicles. In other words, for any EPP (except the first), site occupancy might be high (short dead times) and $p$ low, or vice versa. Nevertheless, we examined the model in two additional scenarios. In each case, we assumed that the dead time is constant. In the first test, we assumed that the dead time was equal to the sigmoid half-time obtained from fitting the quantum content curve. Knowing the initial RRP size (Fig. $6 E$ ), the quantum content $m$ for each EPP, and the recruitment time, we could then iteratively calculate release probability $p$ and number of occupied sites $n$ for each shock (see Materials and Methods), using the 
A

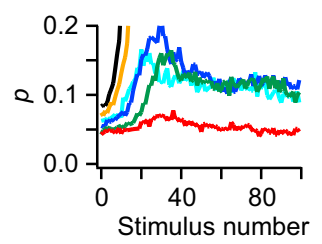

C

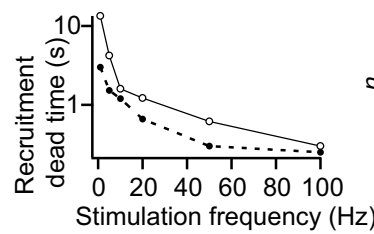

$\mathrm{B}$

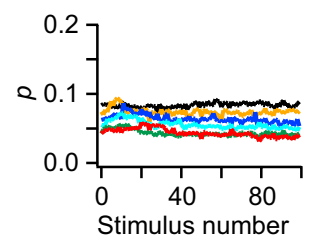

D

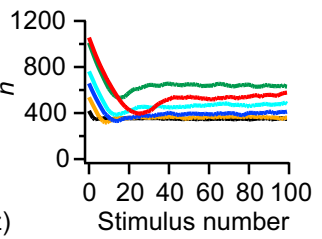

Figure 8. Calculations of $n$ and $p$ during a train of stimuli. Iterative calculations for each EPP during a train, based on different dead times, that is, the interval between exocytosis and reloading of a release site (see Materials and Methods for details). Dead time was constant during any single train. $\boldsymbol{A}$, Dead time was assumed to equal the half-time of the sigmoidal recruitment curve (Fig. 6C), and the release probability $p$ that would be required to produce the observed quantum content for each stimulus is plotted against stimulus number for different frequencies [1 (black), 5 (yellow), 10 (light blue), 20 (dark blue), 50 (green), and 100 (red) Hz]. Some $p$ values rose to improbably high values (sometimes exceeding 1.0). $\boldsymbol{B}$, The same plots shown for dead times that gave minimal variation in $p$ during the train. $\boldsymbol{C}$, The dead times from $\boldsymbol{A}$ (open circles) and $\boldsymbol{B}$ (filled circles) are plotted against stimulus frequency. They show a similar trend and reach a minimum dead time of $250-300 \mathrm{~ms}$ at $100 \mathrm{~Hz}$. D , Values of the number of occupied release sites $(n)$ for each frequency, calculated using the dead time values and $p$ values from $\boldsymbol{B}$ (filled circles).

binomial relationship $m=n \cdot p$ (Del Castillo and Katz, 1954). The results (Fig. $8 \mathrm{~A}$ ) were not satisfying, as calculated $p$ values rose to improbable (and in some cases impossible) levels. As an alternative, we determined the dead time for each stimulus frequency that gave the smallest variation in $p$ during the train, reasoning that release probability is determined mainly by nanodomain calcium concentration, which is determined mostly by calcium currents through voltage-gated calcium channels (and is therefore relatively constant), with a modest contribution from global calcium concentration (Zucker and Regehr, 2002; Neher and Sakaba, 2008), which is also constant during the train (David and Barrett, 2000). Figure $8 B$ shows the resulting $p$ values, which are quite constant for each frequency. Figure $8 C$ shows that these recruitment times (filled circles) were similar to, but faster than the sigmoid half-times (open circles). Part of this difference could reflect the time needed to wake up the sleepy AZs during the beginning of a high-frequency train. Finally, values of $n$ (the total number of occupied release sites) are shown in Figure $8 D$; they were calculated using the $p$ values from Figure $8 B$. At no frequency was depletion $>50 \%$, which seems like a large reserve, although if recruitment were to fail, $n$ would fall to zero in $<1 \mathrm{~s}$ at stimulus frequencies of $10 \mathrm{~Hz}$ and higher.

\section{Discussion}

We have combined two relatively simple observations- the morphological estimate of the total number of synaptic vesicles docked at AZs in resting motor nerve terminals of the adult mouse levator auris muscle, and electrophysiological measurements of the number of quanta of ACh released during repetitive stimulation-in a model of sequential depletion and refilling of the RRP of synaptic vesicles. The morphology was based on fluorescence imaging of fixed terminals labeled with antibodies to Bassoon or Piccolo, two molecules identified as AZ components

(Cases-Langhoff et al., 1996; tom Dieck et al., 1998; Fenster et al., 2000). The staining revealed crisp, round, diffraction-limited spots, localized over postsynaptic folds, as has been described in other synapses, with a density characteristic of AZs (Fukunaga et al., 1983). This pattern was not altered by intense stimulation but was severely disrupted by treatment with proteolytic enzymes. Together, these observations make it reasonable to conclude that the spots mark AZs.

With $\sim 850$ AZs per terminal, each with 2 primary docked vesicles (Nagwaney et al., 2009), there should be in a resting terminal $\sim 1700$ primed vesicles, docked at AZs, presumably composing the RRP. During stimulation at $100 \mathrm{~Hz}$, EPP amplitudes decay exponentially to a plateau. We modeled this (ignoring the initial facilitation) as depletion of the original population, followed after a delay of $\sim 200 \mathrm{~ms}$ by vesicle recruitment, which arrests (and sometimes transiently reverses) the decline of EPP amplitudes. When the train ends, the empty sites are refilled, but not as quickly as during the train; the time constant of recovery after the train was $\sim 800 \mathrm{~ms}$ (vs $\sim 250 \mathrm{~ms}$ during a $100 \mathrm{~Hz}$ train). Because vesicle recruitment has been shown to be calcium dependent (Neher and Sakaba, 2008), the slowdown in refilling after the train ends probably reflects the rapid fall in global calcium that occurs after the train (David and Barrett, 2000). More specifically, in the calyx of Held, which is approximately the same size as the NMJ, the recruitment rate for intracellular calcium concentrations in the range of $0.5-1 \mu \mathrm{M}$ is $0.5-2$ vesicles $/ \mathrm{ms}$. At the NMJ, with an RRP of 1700 vesicles and assuming 50\% depletion during the plateau, 850 vesicles must be recruited. At a rate of 1 vesicle/ms (Neher and Sakaba, 2008), the refilling would require $800 \mathrm{~ms}$, in good agreement with the recovery time constant (823 ms).

Stimulation with trains of different frequencies revealed additional features of the RRP. In particular, we were surprised to find that estimated RRP size varied with stimulus frequency. At $1 \mathrm{~Hz}$ stimulation, the calculated RRP size was less than one-half the calculated RRP size at $100 \mathrm{~Hz}$. The simplest explanation of this effect is that at $1 \mathrm{~Hz}$ exocytosis came only from a subset of AZs, with the others not participating at all. This idea of "sleepy" AZs has been proposed before, based on the observation that trial-totrial fluctuations in synaptic signals (low-frequency stimulation) are significantly smaller than expected (Wang et al., 2010), yielding estimates of release probability in excess of 0.5 , much higher than expected if all AZs were to behave the same. We have confirmed this observation in the mouse levator auris preparation (results not shown). A possible explanation can be found in freeze fracture EMs, which reveal that although most AZs are unitary structures $60 \times 80 \mathrm{~nm}$ in diameter, tight clusters comprising multiple copies of the unitary structures are sometimes observed (Fukunaga et al., 1983; Teng et al., 1999). The closely spaced calcium channels in these multimeric AZs might increase nanodomain calcium selectively at these regions. If so, it would suggest that release probability $p$ is lower in single AZs compared with multimeric AZs. In previous studies (Tabares et al., 2007; Gaffield et al., 2009a,b), we mapped sites of exocytosis in the $\mathrm{spH}$ transgenic mouse. At low stimulus rates, fluorescence increases arose from sites spread all over the terminal, which suggests that the subset of AZs that are active at low stimulus rates are not clustered in any particular region of the terminal. As a check, we measured the locations of the largest Bassoon spots and found that they also are scattered over the entire terminal.

During $100 \mathrm{~Hz}$ stimulation, the sleepy active zones are recruited rapidly, judging by the observation that EPP amplitudes decline exponentially after the first two to five stimuli. During 
this early time, EPP amplitudes usually increase (facilitation), and the idea of awakening active zones suggests that the facilitation is at least in part attributable to the recruitment of additional AZs and their attendant vesicle populations.

Rates of release and recruitment, calculated by fitting the kinetic model to observed EPP rundown during repetitive stimulation, were tightly coupled. As stimulus frequency increased over a 100-fold range the rate of depletion of the RRP increased by $>10$-fold, and the rate of recruitment increased by almost exactly the same amount, so that, especially for frequencies $>10$ $\mathrm{Hz}$, the plateau level of release was quite constant. This is in good agreement with the prediction made from observations at the calyx, in which an approximately linear rise in recruitment rate with calcium concentration balances the increased release attributable to the increased frequency of stimulation (Neher and Sakaba, 2008). Raising the temperature or adding phorbol ester selectively increased the quantum content during the EPP train plateau phase, which, fitted by the kinetic model, is interpreted as a selective increase in vesicle recruitment rate. EGTA had the opposite effect (selectively reducing plateau quantum content), as if recruitment rate were slowed.

While estimated recruitment time fell sharply with increased stimulation frequency, the curve flattened at high frequencies at a value of $\sim 300 \mathrm{~ms}$, suggesting a minimum time limit for recruitment. In the calyx, it has been suggested that recruitment can be divided into two distinct phases, "site clearing," which concerns removing a spent vesicle from the AZ, and reoccupancy, which involves the movement of a replacement vesicle into the core of the AZ. Perhaps at the NMJ one of these events (probably reoccupancy) shows steep calcium dependence, whereas the other cannot occur in less than $\sim 300 \mathrm{~ms}$.

At the mouse NMJ, the number of quanta released by a $100 \mathrm{~Hz}$ train $1 \mathrm{~s}$ in duration is $\sim 3000$, equal to all of the vesicles that we estimate are tethered to $\mathrm{AZ}$ material at rest (primary docked vesicles, secondary docked vesicles, and nondocked vesicles attached to AZ material) (Nagwaney et al., 2009). Using longer trains, we looked for kinetic signs of a switch in recruitment from these populations to others, such as untethered vesicles moving to the AZs from regions deeper in the cytoplasm, or from recycled vesicles that had been part of the original population. However, the plateau values of quantum contents during longer trains showed no evident inflection suggestive of such a shift in recruitment population. Instead, there was usually just a slow linear decline (a few percent per second) in EPP quantum content during longer periods of $100 \mathrm{~Hz}$ stimulation.

We do not know the fate of the vesicles after exocytosis. EM tomography reveals that the membrane adjacent to the AZ, where the secondary vesicles are located, is irregularly folded, unlike vast regions farther from the $\mathrm{AZ}$, which are almost perfectly flat. Nagwaney et al. (2009) suggested that the folded membrane might mark sites of endocytosis and therefore that the secondary docked vesicles might be the products of recent endocytosis. This is consistent with results at snake motor nerve terminals, which possess specialized endocytic sites ("endocytic active zones") observed after light-to-moderate stimulation and distributed in a pattern similar to exocytic AZs viewed by freeze fracture EM (Teng et al., 1999). At the frog NMJ, the situation appears to be different, in that retrieved vesicles move away from the membrane, yet remain readily releasable (Richards et al., 2000, 2003; Rizzoli and Betz, 2004). Likewise, vesicles endocytosed by cultured hippocampal neurons move immediately away from the membrane (Hoopmann et al., 2010).
In the past, most optical studies of vesicle recycling at the NMJ have involved intense (unphysiological) stimulation, which evokes massive exocytosis that leads to a process of bulk endocytosis and the formation of prominent cisternae. The normal situation in vivo may be different, involving local recovery of single vesicles via a fast process of limited capacity. In this regard, it may be more appropriate to think of a motor nerve terminal working normally through the operation of hundreds of independent exoendocytic machines, each centered on an AZ. Recycling and vesicle reuse might then be fast, with vesicles retrieved directly adjacent to AZs, raising their probability of recapture by the AZ filaments, rather than dissociating and moving deeper into the cytoplasm. One could even imagine that the tethers could persist even after exocytosis, and thus the vesicle would remain attached to the AZ throughout its entire cycle.

\section{References}

Betz A, Ashery U, Rickmann M, Augustin I, Neher E, Südhof TC, Rettig J, Brose N (1998) Munc13-1 is a presynaptic phorbol ester receptor that enhances neurotransmitter release. Neuron 21:123-136.

Cases-Langhoff C, Voss B, Garner AM, Appeltauer U, Takei K, Kindler S, Veh RW, De Camilli P, Gundelfinger ED, Garner CC (1996) Piccolo, a novel $420 \mathrm{kDa}$ protein associated with the presynaptic cytomatrix. Eur J Cell Biol 69:214-223.

Ceccarelli B, Grohovaz F, Hurlbut WP (1979) Freeze-fracture studies of frog neuromuscular junctions during intense release of neurotransmitter. I. Effects of black widow spider venom and $\mathrm{Ca}^{2+}$-free solutions on the structure of the active zone. J Cell Biol 81:163-177.

Couteaux R, Pécot-Dechavassine M (1970) Synaptic vesicles and pouches at the level of "active zones" of the neuromuscular junction (in French). C R Acad Sci Hebd Seances Acad Sci D 271:2346-2349.

David G, Barrett EF (2000) Stimulation-evoked increases in cytosolic $\left[\mathrm{Ca}^{2+}\right]$ in mouse motor nerve terminals are limited by mitochondrial uptake and are temperature-dependent. J Neurosci 20:7290-7296.

Del Castillo J, Katz B (1954) Quantal components of the end-plate potential. J Physiol 124:560-573.

Elmqvist D, Quastel DM (1965) A quantitative study of end-plate potentials in isolated human muscle. J Physiol 178:505-529.

Fenster SD, Chung WJ, Zhai R, Cases-Langhoff C, Voss B, Garner AM, Kaempf U, Kindler S, Gundelfinger ED, Garner CC (2000) Piccolo, a presynaptic zinc finger protein structurally related to bassoon. Neuron 25:203-214.

Fukunaga H, Engel AG, Lang B, Newsom-Davis J, Vincent A (1983) Passive transfer of Lambert-Eaton myasthenic syndrome with IgG from man to mouse depletes the presynaptic membrane active zones. Proc Natl Acad Sci U S A 80:7636-7640.

Fukuoka T, Engel AG, Lang B, Newsom-Davis J, Prior C, Wray DW (1987) Lambert-Eaton myasthenic syndrome: I. Early morphological effects of IgG on the presynaptic membrane active zones. Ann Neurol 22:193-199.

Gaffield MA, Betz WJ (2007) Synaptic vesicle mobility in mouse motor nerve terminals with and without synapsin. J Neurosci 27:13691-13700.

Gaffield MA, Tabares L, Betz WJ (2009a) The spatial pattern of exocytosis and post-exocytic mobility of synaptopHluorin in mouse motor nerve terminals. J Physiol 587:1187-1200.

Gaffield MA, Tabares L, Betz WJ (2009b) Preferred sites of exocytosis and endocytosis colocalize during high- but not lower-frequency stimulation in mouse motor nerve terminals. J Neurosci 29:15308-15316.

Heuser JE, Reese TS, Landis DM (1974) Functional changes in frog neuromuscular junctions studied with freeze-fracture. J Neurocytol 3:109-131.

Heuser JE, Reese TS, Dennis MJ, Jan Y, Jan L, Evans L (1979) Synaptic vesicle exocytosis captured by quick freezing and correlated with quantal transmitter release. J Cell Biol 81:275-300.

Hoopmann P, Punge A, Barysch SV, Westphal V, Bückers J, Opazo F, Bethani I, Lauterbach MA, Hell SW, Rizzoli SO (2010) Endosomal sorting of readily releasable synaptic vesicles. Proc Natl Acad Sci USA 107:19055-19060.

Hori T, Takai Y, Takahashi T (1999) Presynaptic mechanism for phorbol ester-induced synaptic potentiation. J Neurosci 19:7262-7267.

Landis DM, Hall AK, Weinstein LA, Reese TS (1988) The organization of 
cytoplasm at the presynaptic active zone of a central nervous system synapse. Neuron 1:201-209.

McLachlan EM, Martin AR (1981) Non-linear summation of end-plate potentials in the frog and mouse. J Physiol 311:307-324.

Millar AG, Bradacs H, Charlton MP, Atwood HL (2002) Inverse relationship between release probability and readily releasable vesicles in depressing and facilitating synapses. J Neurosci 22:9661-9667.

Nagwaney S, Harlow ML, Jung JH, Szule JA, Ress D, Xu J, Marshall RM, McMahan UJ (2009) Macromolecular connections of active zone material to docked synaptic vesicles and presynaptic membrane at neuromuscular junctions of mouse. J Comp Neurol 513:457-468.

Neher E, Sakaba T (2008) Multiple roles of calcium ions in the regulation of neurotransmitter release. Neuron 59:861-872.

Nishimune H, Sanes JR, Carlson SS (2004) A synaptic laminin-calcium channel interaction organizes active zones in motor nerve terminals. Nature 432:580-587.

Nystrom RR, Ko CP (1988) Disruption of active zones in frog neuromuscular junctions following treatment with proteolytic enzymes. J Neurocytol 17:63-71.

Pan B, Zucker RS (2009) A general model of synaptic transmission and short-term plasticity. Neuron 62:539-554.

Rash JE, Ellisman MH (1974) Studies of excitable membranes. I. Macromolecular specializations of the neuromuscular junction and the nonjunctional sarcolemma. J Cell Biol 63:567-586.

Rhee JS, Betz A, Pyott S, Reim K, Varoqueaux F, Augustin I, Hesse D, Südhof TC, Takahashi M, Rosenmund C, Brose N (2002) Beta phorbol esterand diacylglycerol-induced augmentation of transmitter release is mediated by Munc13s and not by PKCs. Cell 108:121-133.

Richards DA, Guatimosim C, Betz WJ (2000) Two endocytic recycling routes selectively fill two vesicle pools in frog motor nerve terminals. Neuron 27:551-559.

Richards DA, Guatimosim C, Rizzoli SO, Betz WJ (2003) Synaptic vesicle pools at the frog neuromuscular junction. Neuron 39:529-541.

Rizzoli SO, Betz WJ (2004) The structural organization of the readily releasable pool of synaptic vesicles. Science 303:2037-2039.
Ruiz R, Casañas JJ, Südhof TC, Tabares L (2008) Cysteine string proteinalpha is essential for the high calcium sensitivity of exocytosis in a vertebrate synapse. Eur J Neurosci 27:3118-3131.

Schneggenburger R, Meyer AC, Neher E (1999) Released fraction and total size of a pool of immediately available transmitter quanta at a calyx synapse. Neuron 23:399-409.

Shapira R, Silberberg SD, Ginsburg S, Rahamimoff R (1987) Activation of protein kinase C augments evoked transmitter release. Nature 325:58 -60.

Stevens CF, Williams JH (2007) Discharge of the readily releasable pool with action potentials at hippocampal synapses. J Neurophysiol 98:3221-3229.

Tabares L, Ruiz R, Linares-Clemente P, Gaffield MA, Alvarez de Toledo G, Fernandez-Chacón R, Betz WJ (2007) Monitoring synaptic function at the neuromuscular junction of a mouse expressing synaptopHluorin. J Neurosci 27:5422-5430.

Teng H, Cole JC, Roberts RL, Wilkinson RS (1999) Endocytic active zones: hot spots for endocytosis in vertebrate neuromuscular terminals. J Neurosci 19:4855-4866.

tom Dieck S, Sanmartí-Vila L, Langnaese K, Richter K, Kindler S, Soyke A, Wex H, Smalla KH, Kämpf U, Fränzer JT, Stumm M, Garner CC, Gundelfinger ED (1998) Bassoon, a novel zinc-finger CAG/glutamine-repeat protein selectively localized at the active zone of presynaptic nerve terminals. J Cell Biol 142:499-509.

Wadel K, Neher E, Sakaba T (2007) The coupling between synaptic vesicles and $\mathrm{Ca}^{2+}$ channels determines fast neurotransmitter release. Neuron 53:563-575.

Wang X, Pinter MJ, Rich MM (2010) $\mathrm{Ca}^{2+}$ dependence of the binomial parameters $p$ and $n$ at the mouse neuromuscular junction. J Neurophysiol 103:659-666.

Waters J, Smith SJ (2000) Phorbol esters potentiate evoked and spontaneous release by different presynaptic mechanisms. J Neurosci 20:7863-7870.

Zhai RG, Bellen HJ (2004) The architecture of the active zone in the presynaptic nerve terminal. Physiology (Bethesda) 19:262-270.

Zucker RS, Regehr WG (2002) Short-term synaptic plasticity. Annu Rev Physiol 64:355-405. 